\title{
Cosmic Jerk, Snap and Beyond
}

\author{
Maciej Dunajski, Gary Gibbons ${ }^{\dagger}$ \\ Department of Applied Mathematics and Theoretical Physics \\ University of Cambridge \\ Wilberforce Road, Cambridge CB3 OWA, UK
}

\begin{abstract}
We clarify the procedure for expressing the Friedmann equation in terms of directly measurable cosmological scalars constructed out of higher derivatives of the scale factor. We carry out this procedure for pure dust, Chaplygin gas and generalised Chaplygin gas energy-momentum tensors. In each case it leads to a constraint on the scalars thus giving rise to a test of General Relativity. We also discuss a formulation of the Friedmann equation as unparametrised geodesic motion and its connection with the Lagrangian treatment of perfect fluids coupled to gravity.
\end{abstract}

\section{Introduction}

It is a striking and slightly puzzling fact that almost all current cosmological observations can be summarised by the simple statement [1, 2, 12]

The jerk of the universe equals one.

The aim of this paper is to uncover the mathematical reason behind this fact and explore some of its consequences, and generalisations.

Our present universe appears to be well described by the Friedmann-Lemaitre-Robertson-Walker (FLRW) metric

$$
g=-d t^{2}+a(t)^{2} h,
$$

where the function $a=a(t)$ is the scale factor, $h$ is a metric on $H^{3}, \mathbb{R}^{3}$ or $S^{3}$ with constant curvature $k=-1,0$ or 1 respectively and the speed of light has been set to 1 .

One may define four cosmological scalars by

$$
H=\frac{1}{a} \frac{d a}{d t}, \quad q=-a\left(\frac{d a}{d t}\right)^{-2} \frac{d^{2} a}{d t^{2}}, \quad Q=a^{2}\left(\frac{d a}{d t}\right)^{-3} \frac{d^{3} a}{d t^{3}}, \quad X=a^{3}\left(\frac{d a}{d t}\right)^{-4} \frac{d^{4} a}{d t^{4}} .
$$

These scalars are known as Hubble, deceleration, jerk and snap respectively 1 . The last three of these are dimensionless and the Hubble has the dimension of inverse of time. The scalars can in principle

\footnotetext{
${ }^{*}$ m.dunajski@damtp.cam.ac.uk

${ }^{\dagger}$ G.W.Gibbons@damtp.cam.ac.uk

${ }^{1}$ The analogous expressions involving 5th and 6th derivatives of $a$ are known as crackle and pop. This terminology goes back to a 1932 advertisement of Kellogg's Rice Crispies which 'merrily snap, crackle, and pop in a bowl of milk'.
} 
be measured using red-shift data and the Hubble law [7, 8, 4, 12, 1, 2, 13]. They arise, for example, as the first four terms in the Taylor expansion of the red-shift $1+z=a\left(t_{o}\right) / a\left(t_{e}\right)$ observed at time $t=t_{o}$ as a function of time of emission $t=t_{e}$. It is important to stress that the Einstein equations have played no role so far. The form of the FLRW metric is derived from symmetry consideration which requires no knowledge of the dynamical variable $a(t)$. The Hubble law is an observational fact also independent of the field equations.

In this paper we shall regard the Einstein equations, in the form of Friedmann equation for $a=a(t)$, as one algebraic constraint between the scalars (1.2). This links the measurement of the cosmological scalars to a test of General Relativity, or any of its modifications (which would lead to different constraints). If one assumes that Einstein equations hold then measuring the cosmological scalars could determine the the equation of state in relating the energy density and the momentum in the perfect fluid energy momentum tensor. We aim to clarify this procedure (which appears to have been initiated in [7] and developed by [8] and is closely related to the Statefinder approach of [12]) and link it to some recent work [3] on the metrisability of projective structures. For example it turns out that the constant jerk condition $Q=1$ is equivalent to Einstein equations with $k=0$ and the dust stress tensor. Using the scaling and translational symmetries of the ODE $Q=1$ allows to put the general solution in the form

$$
a(t)=\sinh ^{\frac{2}{3}}\left(\sqrt{\frac{3 \Lambda}{4}} t\right)
$$

where $\Lambda$ is the cosmological constant. Another motivation comes from quantum cosmology. In the Hamiltonian treatment of general relativity one regards the Einstein equation as a dynamical system equivalent to a forced geodesic motion on an infinite dimensional space $\mathcal{M}$ of three-dimensional Riemannian metrics on initial data surface $\Sigma$. The metric on $\mathcal{M}$ is the DeWitt metric

$$
\mathcal{G}_{g}(\delta g, \delta g)=\int_{\Sigma} \sqrt{g}\left(\operatorname{Tr}\left(\delta g^{2}\right)-\operatorname{Tr}(\delta g)^{2}\right) d^{3} x
$$

(where $\delta g \in T_{g} \mathcal{M}$ and the traces are taken with respect to $g$ ) and the potential is given by the scalar curvature of the Riemannian metric on $\Sigma$. See e.g. [5] where this is discussed. In the context of quantum cosmology this analysis needs to be modified in the presence of matter and cosmological constant. In Section 3 we shall use a different point of view to relate the Friedmann equation a geodesic motion on two-dimensional surface of revolution. In Section 4 we shall make contact with the Lagrangian treatment of perfect fluids coupled to gravity. The problem of finding a metric whose unparametrised geodesics coincide with the integral curves of a second order ODE is summarised in the Appendix.

\section{Cosmological scalars and the Friedmann equation}

Consider the Einstein equations with cosmological constant $\Lambda$ and matter described by the perfect fluid energy momentum tensor

$$
T_{\mu \nu}=(\rho+p) U_{\mu} U_{\nu}+p g_{\mu \nu}
$$

where $U_{\mu}=\nabla_{\mu} t, \rho=\rho(t)$ describes the matter density and $p=p(t)$ is the pressure. The energymomentum conservation yields

$$
a \frac{d \rho}{d t}+3(\rho+p) \frac{d a}{d t}=0
$$

and the Einstein equations reduce to the Friedmann equation

$$
\left(\frac{d a}{d t}\right)^{2}+k=\frac{8 \pi G}{3} \rho a^{2}+\frac{\Lambda}{3} a^{2}
$$


Pure dust. Consider the pressure-free matter $p=0$. The energy conservation gives $\rho a^{3}=M$ where $M$ is a constant. Let us now consider a system of three equations consisting of (2.4) and its first two time derivatives. We regard this as a system of algebraic equations for the constants $(k, \Lambda, G M)$ which can therefore be expressed as functions of $(a, \dot{a}, \ddot{a}, \dddot{a})$. Take the third derivative of (2.4) and substitute the expressions for $(k, \Lambda, G M)$. The resulting equation 2 does not contain any parameters and can be expressed in terms of the cosmological scalars (1.2) as

$$
X+2(q+Q)+q Q=0 .
$$

This fourth order ODE is equivalent to the Friedmann equation and has an advantage that it appears as a constraint on directly measurable quantities. Thus it provides the test of the model alluded to in the Introduction. If only two constants $(\Lambda, G M)$ are eliminated between (2.4) and its first derivative then the second derivative of (2.4) yields

$$
k=a^{2} H^{2}(Q-1)
$$

where $k$ is regarded as a parameter. This is the formula obtained in [7]. In particular if $k=0$ this relation reduces to a third order ODE

$$
Q=1 \text {. }
$$

This constant jerk condition is consistent with recent redshift analysis which provides the lower bound for the jerk implying that $Q>0$. Compare a related discussion in [13].

Chaplygin Gas. This exotic form of matter is given by the perfect fluid energy momentum tensor with the equation of state

$$
p=-\frac{A}{\rho}
$$

where $A$ is a positive constant. In the cosmological context this model was proposed in [9]. The negative pressure allows to describe a transition from a decelerated universe to cosmic acceleration. The energy conservation equation gives

$$
\rho=\sqrt{A+\frac{B}{a^{6}}}
$$

For small $a(t)$ this reduces to the dust cosmology $\rho=\sqrt{B} a^{-3}$, and for large $a$ one gets the de Sitter Universe $\rho=\sqrt{A}, p=-\sqrt{A}$. In between these two regimes one can use the expanded expression

$$
\rho=\sqrt{A}+\frac{B}{2 \sqrt{A}} a^{-6} .
$$

Thus $\sqrt{A}$ plays the role of a cosmological constant. We insert this to the Friedmann equation with $\Lambda=0$ and follow the procedure of eliminating the constants by differentiation. This leads to an approximate constraint

$$
X+5(Q+q)+q Q=0
$$

\footnotetext{
${ }^{2}$ This idea of eliminating parameters and reinterpreting them as constants of integration has a long history. It goes back at least to Halphen [6] who obtained a fifth order ODE characterising conics in $\mathbb{R P}^{2}$. In the inhomogeneous coordinates $(a, t)$ the five parameter family of conics is

$$
a^{2}=c_{1} t^{2}+c_{2} a t+c_{3} a+c_{4} t+c_{5}
$$

Eliminating the parameters $\left(c_{1}, \ldots, c_{5}\right)$ between this equation and its fourth derivatives and substituting in the fifth derivative yields the ODE

$$
\frac{d^{3}}{d t^{3}}\left(\left(\frac{d^{2} a}{d t^{2}}\right)^{-\frac{2}{3}}\right)=0
$$
}


which is valid when $a^{-6} B / 2 \sqrt{A}$ is small compared to the unity.

Generalised Chaplygin Gas. This is the generalisation of the previous case, where

$$
p=-\frac{A}{\rho^{\alpha}}, \quad 0 \leq \alpha \leq 1
$$

Introducing an additional constant $\alpha$ allows a description of a universe evolving from the pure dust matter to a cosmological constant with an intermediate epoch with non-zero cosmological constant and perfect fluid matter satisfying the equation of state $p=\alpha \rho$.

The energy conservation gives

$$
\rho=\left(A+B a^{-3(\alpha+1)}\right)^{\frac{1}{\alpha+1}} \approx A^{\frac{1}{\alpha+1}}+\frac{A^{\frac{1}{\alpha+1}}}{\alpha+1} \frac{B}{A} a^{-3(\alpha+1)}+\ldots .
$$

Inserting the expanded formula into the Friedmann equation with $\Lambda=0$ leads to an expression with three arbitrary constants. Our procedure gives the constraint

$$
X+(3 \alpha+2)(Q+q)+q Q=0
$$

in agreement with (2.5) and (2.6) when $\alpha=0$ and $\alpha=1$ respectively. We may however want to eliminate $\alpha$ from this equation by another differentiation. This requires an additional cosmological scalar crackle

$$
Y=a^{4}\left(\frac{d a}{d t}\right)^{-5} \frac{d^{5} a}{d t^{5}}
$$

A rather complicated (MAPLE) calculation leads to a relatively simple constraint

$$
-2 q X-2 Q q^{2}-Y q-2 X Q-3 X q^{2}-Q^{2} q-Y Q+X^{2}-3 q^{3} Q-q X Q+Q^{3}-2 Q^{2} q^{2}=0
$$

which should hold if Einstein equations with the generalised Chaplygin gas energy momentum holds. This constraint is again approximate and is valid only in the regime where the higher order terms in the expansion of $\rho$ can be dropped.

A different constraint involving $Y$ appeared in the work of Hut [8] who considered a mixture of perfect dust and radiation in the energy momentum tensor.

\section{Friedmann equations as geodesic motion}

In this section we shall relate the Friedmann equation to a geodesic motion on a surface of revolution with metric given explicitly by (3.8).

For simplicity we consider the pure dust case $p=0$. Eliminating the constant $G M$ between the Friedmann equation and its time derivative yields a second order ODE

$$
\frac{d^{2} a}{d t^{2}}=\frac{1}{2}\left(\Lambda a-\frac{k}{a}\right)-\frac{1}{2 a}\left(\frac{d a}{d t}\right)^{2} .
$$

Can we think of the integral curves of this equation as unparametrised geodesics of some metric on a surface with local coordinates $(a, t)$ ? This question can be asked about any second order ODE of the form $\ddot{a}=F(t, a, \dot{a})$. The necessary condition is that $F$ is at most cubic in the first derivatives $\dot{a}$. This condition (which was known to R. Liouville [10]) is obviously satisfied by (3.7). Therefore the integral curves of (3.7) are geodesics of some projective structure (an equivalence class of torsion-free connections sharing the same unparametrised geodesics). In [10] Liouville also began the study of sufficient conditions for a projective structure to come from a metric, but these were derived only 
recently [3]. They come down to vanishing of three weighted invariants of differential order at most 8 in connection defining the projective structure. We have checked that these invariants vanish of (3.7) and so there exist an underlying metric. Following a linear algorithm lied down in [10] and summarised in the Appendix one can construct the metric explicitly. The answer is

$$
\mathcal{G}=\frac{d t^{2}}{k a-\frac{\Lambda}{3} a^{3}+c}+\frac{a d a^{2}}{\left(k a-\frac{\Lambda}{3} a^{3}+c\right)^{2}},
$$

where $c$ is a constant equal to a negative multiple of the Newton's constant. To verify this, write the geodesic equations for (3.8) and eliminate the affine parameter between the two equations thus expressing $a$ as a function of $t$. This will lead back to (3.7). The scalar curvature of the metric (3.8) is

$$
\frac{2 k \Lambda a^{3}+9 \Lambda a^{2} c+6 k^{2} a+3 k c}{6 a^{2}}
$$

Thus the metric has constant curvature (and therefore it is projectively flat) if and only if $k=0$. In this case there exist a coordinate transformation $(t, a) \rightarrow(\hat{t}(t, a), \hat{a}(t, a))$ such that, in the new coordinates the Friedmann equations are

$$
\frac{d^{2} \hat{a}}{d \hat{t}^{2}}=0
$$

The cubic denominators in (3.8) have real zeroes, and so the metric may appear singular. In fact these zeroes correspond to coordinate singularities, and are not present in curvature scalars. For example when $k=0$ the curves $a^{3}=3 c / \Lambda$ are only apparent singularities as the metric has constant curvature.

\section{Lagrangian description of perfect fluids}

In this section we shall make contact with a standard Lagrangian treatment of perfect fluids coupled to gravity [11] and then specialise to the FLRW case. We shall then use this formalism to derive the geodesic formulation analogous to (3.8) when radiation and other forms of matter are present in the energy-momentum tensor.

We shall restrict our general discussion to the case of an irrotational (i. e. vorticity-free) perfect fluid. We introduce a potential $\psi$ and let

$$
Z=-g^{\mu \nu} \nabla_{\mu} \psi \nabla_{\nu} \psi
$$

We take as Lagrangian for $\psi$

$$
L=L(Z)
$$

The energy momentum tensor is

$$
T_{\mu \nu}=2 L_{Z} \nabla_{\mu} \psi \nabla_{\nu} \psi+L g_{\mu \nu} .
$$

Comparing this with a perfect fluid (2.3) with $U_{\mu}=\left(\nabla_{\mu} \psi\right) / Z$ we may identify

$$
\rho=2 Z L_{Z}-L \quad p=L .
$$

Adding a constant to the Lagrangian $L(Z)$ introduces the cosmological term in the FLRW models:

$$
L \rightarrow L+\gamma \quad \rho \rightarrow \rho-\gamma, \quad p \rightarrow p+\gamma, \quad T_{\mu \nu} \longrightarrow T_{\mu \nu}+\gamma g_{\mu \nu}
$$

- If $p=\alpha \rho$ then $L=Z^{\frac{1+\alpha}{2 \alpha}}$. 
- For a Born-Infeld scalar field

$$
L=-\sqrt{1-Z}+1
$$

we get

$$
p=\frac{\rho}{1+\rho} .
$$

Omitting the one, which amounts to changing the zero of the energy scale, or cancelling a cosmological term, gives the Chaplygin gas

$$
p=-\frac{1}{\rho}
$$

mentioned earlier.

The shift symmetry $\psi \rightarrow \psi+$ constant gives rise to a conserved current which, in this perfect fluid context, may be interpreted as the entropy current.

$$
s^{\mu}=-\frac{\partial L}{\partial\left(\nabla_{\mu} \psi\right)}, \quad \nabla_{\mu} s^{\mu}=0 .
$$

As an example, consider radiation $L=Z^{2}$. The equations of motion are

$$
\nabla_{\mu}\left((\nabla \psi)^{2} \nabla^{\mu} \psi\right)=0
$$

or, as long as $\nabla^{\mu} \psi$ is time-like

$$
\left(g^{\mu \nu}-2 U^{\mu} U^{\nu}\right) \nabla_{\mu} \nabla_{\nu} \psi=0 .
$$

Two simple solutions in flat space-time are

- $\psi=f(t-z)$, where $z$ is one of the spatial coordinates and $f$ is an arbitrary function. This gives a trivial solution with vanishing energy momentum tensor.

- $\psi=t$. This represents a uniform fluid at rest. Linear perturbations about this, or indeed any, solution are easily seen to travel with respect to it with speed $\frac{1}{\sqrt{3}}$.

Interestingly the action, and hence the equations of motion, are invariant under Weyl conformal rescalings

$$
\psi \rightarrow \psi, \quad g_{\mu \nu} \rightarrow \Omega^{2}(x) g_{\mu \nu} .
$$

In other words the equations (4.9) are conformally invariant, just as is Yang-Mills theory in four space-time dimensions.

\subsection{Coupling to FLRW models}

To couple to gravity we would consider the Lagrangian ( up to a boundary term)

$$
\int\left(\frac{R}{16 \pi G}+L(Z)\right) \sqrt{-g} d^{4} x .
$$

To obtain the full consequences of the Einstein equations we substitute the ansatz $\psi=\psi(t)$ and

$$
g=-N^{2} d t^{2}+a(t)^{2} h,
$$

where $N=N(t)$ is an arbitrary lapse function into the action (4.10). We vary with respect to $\psi, a$ and $N$. The first two Euler-Lagrange equations are second order in time. The third variation gives 
a constraint on the initial values $\psi, \dot{\psi}$ and $a, \dot{a}$ which is consistently evolved by the two second order equations. It is convenient to make the choice $N=1$ after variation. It then follows that the constraint is just the vanishing of the Hamiltonian $\mathcal{H}$ obtained from the Lagrangian

$$
\mathcal{L}=\frac{a^{3}}{4} L(Z)-\frac{3}{8 \pi G}\left(a(\dot{a})^{2}-k a\right), \quad \text { where } \quad Z=\dot{\psi}^{2}
$$

by a standard Legendre transform. One may check that the resulting system of ordinary differential equations is the same as the system one would obtain by substitution of the ansatz (4.11) into the full Einstein equations.

Clearly, for a general equation of state $p=p(\rho)$, the kinetic term in the Lagrangian for $\psi$ will not be quadratic in $\dot{\psi}$ and hence the standard Jacobi procedure will not lead to a metric. The exceptional case is of course 'stiff matter', $p=\rho$ which is well known to be represented by a standard massless scalar field. We shall instead use the procedure introduced in Section 3 to construct a metric whose geodesics coincide with unparametrised extremals of the Euler-Lagrange equations of (4.12).

The conservation of momentum $a^{3} \dot{\psi} L^{\prime}(Z)=$ const can be used to find $\dot{\psi}=\dot{\psi}(a)$. It is convenient to introduce a function $f=f(a)$ such that

$$
\frac{d f}{d a}=2 \pi G a^{2} L(Z(a))+k
$$

The equations of motion resulting from (4.12) become

$$
\frac{d^{2} a}{d t^{2}}=-\frac{1}{2 a} \frac{d f}{d a}-\frac{1}{2 a}\left(\frac{d a}{d t}\right)^{2} .
$$

Examining the obstructions of [3] shows that the projective structure defined by (4.13) is metrisable for any $f(a)$ (and therefore for any choice of the Lagrangian $L$ ). Following the algorithm of [3] leads to the expression for the metric

$$
\mathcal{G}=\frac{d t^{2}}{f(a)}+\frac{a d a^{2}}{f(a)^{2}} .
$$

Unparametrised geodesics of this metric are integral curves of (4.13). For example if $p=\alpha \rho$ and the cosmological constant is allowed then $L(Z)=Z^{\frac{1+\alpha}{2 \alpha}}+\gamma$ and

$$
f(a)=-\frac{2 \pi \alpha G}{3 \alpha+1}\left(\frac{b}{1+\alpha}\right)^{1+\alpha} a^{-3 \alpha-1}+\frac{2 \pi G \gamma}{3} a^{3}+k a+c .
$$

where $b$ and $c$ are some constants. The pure dust case considered in Section 3 is recovered in the limit $\alpha \rightarrow 0$. In this case $f$ is cubic in $a$ and the metric (4.14) becomes (3.8) if $\gamma=-\Lambda /(2 \pi G)$.

For radiation $(\alpha=1 / 3)$ the Legendre transform gives the Hamiltonian and the momenta

$$
\begin{aligned}
\mathcal{H} & =\frac{3 a^{3}}{4}(\dot{\psi})^{4}-\frac{3}{8 \pi G}\left(a(\dot{a})^{2}+k a\right) \\
p_{\psi} & =a^{3}(\dot{\psi})^{3} \\
p_{a} & =-\frac{3}{4 \pi G} a^{2} H
\end{aligned}
$$

where $H=\dot{a} / a$. Note that the conserved momentum $p_{\psi}$ is essentially the conserved entropy of the radiation (i.e. number of photons). The phase $\psi$ is ignorable both in the technical and the vernacular sense since it disappears from the Hamiltonian. 


\section{Summary}

We have studied the Friedmann equation from the point of view of geometry of ODEs.

In Section 2 we set up an algorithm replacing the 2nd order Friedmann equation which involves a number of parameters (this number depends on a choice of equation of state) by a constraint on directly observable cosmological scalars. The validity of resulting constraints can in principle be verified by observations, thus providing the experimental test of General Relativity as well as rulling out some equations of state. For example the Friedmann equation with $k=0$ and the dust energy-momentum tensor is equivalent to the constant jerk condition $Q=1$.

The constraints discussed in Section 2 give rise to curves in the $(a, t)$ plane, one curve through each point in each direction, and in Sections 3 and 4 we have demonstrated that these curves are unparametrised geodesics of some two-dimensional (pseudo)-Riemannian metric. This is an alternative to the more usual DeWitt approach and may play a role in projective quantisation of Cosmology which does not refer to a specific (time) parametrisation of classical trajectories.

\section{Appendix}

Recall that a projective structure on an open set $U \subset \mathbb{R}^{2}$ is an equivalence class of torsion free connections on $T U$. Two connections are projectively equivalent if they share the same unparametrised geodesics. Let $(a, t)$ be local coordinates on $U$. Eliminating a parameter $s$ between the two geodesic equations $\ddot{x}^{i}+\Gamma_{j k}^{i} \dot{x}^{j} \dot{x}^{k}=v \dot{x}^{i}$ where $x^{i}(s)=(t(s), a(s))$ yields a second order ODE

$$
\frac{d^{2} a}{d t^{2}}=A_{3}(t, a)\left(\frac{d a}{d t}\right)^{3}+A_{2}(t, a)\left(\frac{d a}{d t}\right)^{2}+A_{1}(t, a)\left(\frac{d a}{d t}\right)+A_{0}(t, a)
$$

where the functions $A_{\alpha}$ are given in terms of the coefficients of a connection in a given projective class

$$
A_{0}=-\Gamma_{11}^{2}, \quad A_{1}=\Gamma_{11}^{1}-2 \Gamma_{12}^{2}, \quad A_{2}=2 \Gamma_{12}^{1}-\Gamma_{22}^{2}, \quad A_{3}=\Gamma_{22}^{1} .
$$

Conversely, any ODE of the form (A1) gives rise to a projective structure.

A projective structure is called metrisable if the integral curves of the ODE (A1) are unparametrised geodesics of a metric

$$
g=E(t, a) d t^{2}+2 F(t, a) d t d a+G(t, a) d a^{2} .
$$

In this case the four functions $A_{\alpha}$ are given in terms of $(E, F, G)$ and their first derivatives. The corresponding expressions can be derived by calculating the Levi-Civita connection of $g$ and reading off the $A_{\alpha}$ s from (A2). The substitution

$$
E=\psi_{1} / \Delta^{2}, \quad F=\psi_{2} / \Delta^{2}, \quad G=\psi_{3} / \Delta^{2}, \quad \Delta=\psi_{1} \psi_{3}-\psi_{2}{ }^{2}
$$

linearises these expressions thus leading to a characterisation of the metricity condition as consistency conditions for an overdetermined system of linear PDEs

Theorem 1 (R. Liouville 1889 [10]). A projective structure corresponding to the second order ODE (A1) is metrisable on a neighbourhood of a point $p \in U$ iff there exists functions $\psi_{1}, \psi_{2}, \psi_{3}$ defined on a neighbourhood of $p$ such that

$$
\psi_{1} \psi_{3}-\psi_{2}^{2}
$$


does not vanish at $p$ and such that the equations

$$
\begin{aligned}
\frac{\partial \psi_{1}}{\partial t} & =\frac{2}{3} A_{1} \psi_{1}-2 A_{0} \psi_{2}, \\
\frac{\partial \psi_{3}}{\partial a} & =2 A_{3} \psi_{2}-\frac{2}{3} A_{2} \psi_{3}, \\
\frac{\partial \psi_{1}}{\partial a}+2 \frac{\partial \psi_{2}}{\partial t} & =\frac{4}{3} A_{2} \psi_{1}-\frac{2}{3} A_{1} \psi_{2}-2 A_{0} \psi_{3}, \\
\frac{\partial \psi_{3}}{\partial t}+2 \frac{\partial \psi_{2}}{\partial a} & =2 A_{3} \psi_{1}-\frac{4}{3} A_{1} \psi_{3}+\frac{2}{3} A_{2} \psi_{2}
\end{aligned}
$$

hold on the domain of definition.

This system is overdetermined, as there are more equations than unknowns. In [3] the consistency conditions for this system where found in terms of point invariants of the associated second order ODE (A1). The details of this construction and the invariants themselves are rather complicated and we refer the reader to [3]. The invariants obstructing metricity vanish for the projective structures (3.7) and (4.13) arising in our paper, and the corresponding metrics are found by solving the linear system (A4).

\section{References}

[1] Alam, U., Sahni, V., Saini T. D., \& Starobinsky, A. A. (2003) Exploring the Expanding Universe and Dark Energy using the Statefinder Diagnostic," Mon. Not. Roy. Astron. Soc. 344, 1057.

[2] Blandford, R. D. (2004) Measuring and modeling the universe: A theoretical perspective. in W. L. Freedman (Ed.), Carnegie Observatories Astrophysics Series, Vol. 2: Measuring and Modeling the Universe, 377-388. Cambridge, Cambridge University Press.

[3] Bryant, R. L., Dunajski, M. \& Eastwood, M. (2008) Metrisability of two-dimensional projective structures arXiv:0801.0300v1

[4] Chiba, T. \& Nakamur T. (1998) The luminosity distance, the equation of state, and the geometry of the universe, Prog. Theor. Phys. 100, 1077

[5] Fisher, A. E. \& Marsden, J. E. (1971) The Einstein Equations of Evolution - A Geometric Approach, J. Math. Phys. 13, 546.

[6] Halphen, G. (1879) Sur l'quation diffrentielle des coniques. Bulletin de la Socit Mathmatique de France, 7 83-85.

[7] Harrison, E. R. (1976) Observational tests in cosmology, Nature 260, 591-592.

[8] Hut, P. (1977) Cosmological tests of general relativity, Nature 267, 128-130.

[9] Kamenshchik, A. Y., Moschella, U., \& Pasquier V. (2001) An alternative to quintessence, Phys.Lett. B511 265-268.

[10] Liouville, R. (1889) Sur les invariants de certaines équations différentielles et sur leurs applications, Jour. de l'Ecole Politechnique, Cah.59, 7-76. 
[11] Schutz, B. F. (1970) Perfect Fluids in General Relativity: Velocity Potentials and a Variational Principle, Phys Rev D2 2762-2773.

[12] Sahni, V., Saini T. D., Starobinsky, A. A., \& Alam, U. (2003) Statefinder - a new geometrical diagnostic of dark energy, JETP Lett. 77 201-206.

[13] Visser, M. (2004) Jerk, snap, and the cosmological equation of state, Class. Quant. Grav. 21 2603 